\title{
LA JUSTIFICACIÓN Y LA INFERENCIA EN EL CONOCIMIENTO PERCEPTUAL*
}

En los escritos filosóficos tradicionales es frecuente encontrar las siguientes condiciones como necesarias y suficientes para atribuirle conocimiento a una persona: " $S$ sabe que $p$ " si y sólo si

i) $p$ es verdadera

ii) $S$ cree que $p$

iii) $S$ está justificado en creer que $p$

Aquí me limitaré a examinar la condición de justificación y el papel que desempeña especificamente en las afirmaciones de creencia y conocimiento de tipo perceptual. Basta recordar la interminable discusión entre los filósofos del pasado, que ha cobrado un nuevo interés en la actualidad, acerca de si la percepción y el conocimiento del mundo exterior son inferenciales, para darnos cuenta de la importancia que han tenido la noción de "inferencia", y otras conectadas con ella -como la de la justificación_, en el ámbito de la percepción. A pesar de no existir una caracterización homogénea de la "inferencia", la tendencia general ha sido la de considerar que la percepción es inferencial. No cuestionaré aqué esta tesis sino más bien, para efectos de la discusión, la supondré verdadera. En la discusión subsiguiente, me centraré en una manera de entender dicha afirmación, que es la adoptada por Ayer en prácticamente todos sus escritos filosóficos dedicados al problema de la percepción, y en las conexiones entre la noción de inferencia y la de justificación en dicha postura.

Ayer suscribe la tesis de que los juicios perceptuales son inferenciales, en el sentido de que al hacer cualquier juicio perceptual nos comprometemos con muchas otras afirmaciones que, estrictamente hablando, rebasan lo percibido. Ayer formula la tesis de las tres maneras siguientes:' a) El juicio perceptual rebasa los datos en los que se funda; $b$ ) El juicio perceptual afirma más de lo que está contenido en la experiencia que lo hace surgir; c) El juicio per-

- Agradezco al profesor Mark de Bretton Platts, cuyos comentarios ayudaron en gran medida a mi comprensión de los problemas que aqứ se discuten. 
ceptual hace asunciones que pueden ser falsas y a la vez consistentes con la experiencia. ${ }^{1}$

Estas diferentes formulaciones engloban tres ideas básicas:

1) El juicio perceptual va más allá de lo dado.

2) El juicio perceptual se funda o basa en lo dado.

3) Lo dado da origen al juicio perceptual.

La primera apunta al elemento inferencial de los juicios perceptuales, la segunda a la relación de justificación entre lo dado y el juicio perceptual y la tercera a su relación causal.

De ser cierto que los juicios perceptuales son inferenciales, debe ser posible describir lo estrictamente percibido, y para ello Ayer crea un lenguaje que describe los contenidos de la experiencia del observador. La utilidad que Ayer encuentra en crear dicho lenguaje es que nos permitirá esclarecer y explicitar los elementos en los que se basan nuestros juicios ordinarios de percepción. Ayer establece, ex hypothesi, que la forma de describir lo estrictamente percibido se logra despojando de compromisos ontológicos a los juicios perceptuales. A estos juicios neutros ontológicamente, los llama enunciados de experiencia (en adelante, enunciados-E). La ausencia de compromisos ontológicos se manifiesta, según Ayer, en que para establecer la verdad de los enunciados-E no se requiere que se cumpla ninguna condición de tipo predictivo; esto equivale a decir que, para establecer su verdad, no requieren ni la verdad ni la falsedad de ninguna otra proposición. A diferencia de éstos, la verdad de los juicios perceptuales, al igual que la de todas las demás proposiciones, se establece a partir de la verdad de otras proposiciones. Ayer ilustra de la siguiente manera el sentido en el que los enunciados perceptuales (de aquí en adelante enunciados-P) son inferenciales:

...Sólo necesitamos considerar la cantidad de asunciones que nuestros juicios ordinarios perceptuales conllevan. Para empezar, encontramos las asunciones que, como ya hemos visto, están implicadas en la caracterización de algo, por ejemplo una mesa, como un objeto físico. Éste tiene que ser accesible a más de un sentido y a más de un observador, y debe ser capaz de existir cuando no es percibido. Además, debe de ocupar una posición, o una serie de posiciones, en un espacio tridimensional y continuar existiendo por algún tiempo. Ciertamente puede argüirse que éstas no son, en forma directa, asunciones empíricas, sino postulados de un sistema conceptual. Estos postulados establecen el marco de referencia en el cual se acomodan la mayoría de nuestras observaciones. Sin em-

1 Cf. "Has Austin Refuted the Sense-datum Theory?" recogido en el libro de Ayer, Metaphysics and Common Sense, Macmillan, 1969, p. 131. 
bargo, sigue existiendo la posibilidad de que no se satisfagan en algunas instancias particulares. Los esquimales descubrieron que las imágenes que confundieron con focas, no eran tangibles; la presencia de las víboras que el borracho cree ver no es corroborada por otros observadores, y el hecho de que no sea corroborada se considera como prueba para decir que las viboras imaginadas no pueden existir sin ser percibidas... No es solamente una cuestión acerca de la validez de estas asunciones generales. Nuestros juicios perceptuales rara vez son indefinidos, en el sentido de que afirmemos solamente que percibimos un objeto físico de cierto tipo. Normalmente lo identificamos como un objeto de un tipo específico y esto introduce más asunciones, como por ejemplo, que el objeto es sólido, o flexible, o que no está hueco. Estas asunciones pueden relacionarse con la utilidad del objeto, como cuando lo identificamos como una navaja de bolsillo o un teléfono: pueden relacionarse con su constitución física, como en la identificación de un objeto como una naranja o una manzana, lo cual negaría que estuviera hecho de cera. $^{2}$

También es importante hacer notar que la tesis de la inferencialidad adoptada por Ayer no lo compromete, eo ipso, a decir que los objetos materiales no se perciban directamente, esto es, que la percepción de los objetos materiales sea mediata. Esto es suficiente para que nos demos cuenta de la forma tan idiosincrática en que Ayer usa la noción de inferencia.

Por otro lado, Ayer admite que los enunciados-E sí son inferenciales en el sentido de que, a pesar de que haya una relación directa entre el sujeto y sus experiencias - que son las que describen los enunciados- $\mathrm{E}$ - el sujeto tiene que inspeccionar su experiencia para poder describirla y por tanto, reconocerla. Pero, para ello, el sujeto debe poder discriminar las experiencias y esta capacidad, al igual que otras capacidades humanas, depende de sus experiencias pasadas y del entrenamiento al que ha sido expuesto: lo que el sujeto perciba y cómo lo perciba dependerá tanto de su aprendizaje como de su familiaridad con lo percibido. Sin embargo, según Ayer, conceder esto no afecta el contraste deseado entre tipos de proposiciones, ya que en el caso de los enunciados-E ninguna de estas asunciones rebasaría, ontológicamente, el contenido de las experiencias.

Ayer dice acerca de la relación de inferencia entre los enunciados-E y los enunciados-P que:

a) No se trata de una relación deductiva.

b) La verdad de los enunciados-E no constituye una condición suficiente para la verdad de los enunciados-P.

2 The Central Questions of Philosophy, Weidenfeld and Nicholson, London, 1973, pp. 81-82. 
c) La verdad de los enunciados-E no constituye una condición necesaria para la verdad de los enunciados-P.

d) Los enunciados-P se infieren inductivamente de los enunciados-E.

La razón que presenta Ayer para sostener $a$ ) y $b$ ) es que la verdad del enunciado-E que da lugar al enunciado-P es lógicamente consistente con la falsedad de éste; esto es, que el observador puede tener las experiencias en cuestión sin que se siga que el objeto material que él cree percibir exista, ya que podría haber tenido la misma experiencia aun cuando hubiera estado hipnotizado o hubiera sido víctima de alguna otra ilusión. ${ }^{3}$

Con respecto al punto $c$ ), en donde se niega que los enunciados-E constituyan la condición necesaria para aceptar los enunciados-P, Ayer cree, sin embargo, que la verdad del enunciado-P supone, de alguna manera, la verdad de algún enunciado-E:

...Así como he hablado que los enunciados perceptuales se basan necesariamente en los enunciados de experiencia, no he dicho que los impliquen; la razón es que, dado que no hay una línea muy definida respecto a la clase de experiencias en la cual puede basarse, es imposible decir exactamente cuál es la disyunción de enunciados de experiencias que implica. ${ }^{4}$

Así, Ayer sólo se compromete a decir que algún enunciado-E $\longrightarrow$ o conjunto de enunciados-E - debe satisfacerse; esto es, debe ser verdadero algún enunciado-E $\longrightarrow$ o conjuntamente verdaderos algunos enunciados-E-, si es que el enunciado-P ha de ser verdadero.

En el punto $d$ ), el enunciado-P es, con respecto a los enunciados-E, 10 que las generalizaciones empiricas son con respecto a las instancias particulares de las cuales se obtienen. Ambos casos se asemejan, según Ayer, en que tanto una generalización empírica enumerativa como un enunciado-P son el resultado de un "salto", ya que en ninguno de los dos casos se garantiza la verdad de éstos, pero difieren en cuanto a la forma de explicar el salto. En las generalizaciones empíricas, este se debe a que ningún número de instancias particulares agotaría los casos cubiertos por la generalización. En cambio, en el caso perceptual, ningún número de descripciones-E puede garantizar la verdad del enunciado-P, ya que éste incorpora una afirmación ontológica y -como ya mencioné con anterioridad— las descripciones-E no

3 De esto parecería seguirse que para Ayer habria una ontologla de sucesos mentales, ya que al hacer un juicio acerca de ellos, implicitamente nos comprometemos, al igual que en el caso de los juicios perceptuales, a una serie de asunciones, como por ejemplo, que sólo es percibido por el sujeto, que no son objetos públicos y perdurables, etc.

4 "Has Austin refuted the Sense-datum Theory?", op. cit., p. 147. 
contienen ninguna carga ontológica. Otra diferencia entre la generalización empírica y el caso perceptual es que, en el primero, la inferencia es supuestamente consciente, mientras que en el segundo - según Ayer- no necesita serlo.

Ayer a veces expresa la tesis de la inferencia diciendo que los objetos materiales son construcciones teóricas con respecto a las experiencias:

...Mi versión de la manera como se desarrolla la teoría del sentido común acerca del mundo físico no pretende ser histórica. Los niños, quienes muy rápidamente asumen la teoría, no la elaboran ellos mismos, sino que se les enseña un lenguaje que ya la contiene, y aunque fuera lógicamente posible, es fácticamente improbable que la hubieran adquirido de alguna otra manera. He contado una historia ficticia, cuyo objeto ha sido el de poner de relieve las características generales de nuestra experiencia que hacen que cada uno de nosotros emplee con éxito la teoría. Con el objeto de hacer destacar estas características, he representado lo que en realidad es un proceso de análisis, como un proceso de construcción. En líneas generales, mi descripción de este proceso ha sido muy similar a la de Hume. La diferencia principal es que, mientras él encontró en las relaciones de "constancia y coherencia" que nuestras "percepciones" presentan, una forma de explicar cómo caemos en el error de tratarlas como objetos perdurables, yo he presentado estas relaciones, no para dar razón del engaño, sino para justificar una teoría aceptable. ${ }^{5}$

Esta reconstrucción racional de la génesis de los términos que se refieren a objetos físicos, no es, para Ayer, un proceso estrictamente lógico, sino más bien "un ejercicio de la imaginación". Es claro que, puesto que la inferencia no es deductiva, no puede ser un proceso estrictamente lógico. Sin embargo, esto no nos obliga a decir que sea un proceso psicológico que, de hecho, se dé.

Ahora bien, zcómo se conecta esta tesis de la inferencia con la de la justificación? Antes de responder a esta interrogante, consideraré lo que Ayer dice acerca de la justificación.

\section{II}

Ayer acepta la caracterización tradicional de "conocimiento" en términos de "creencia verdadera justificada", pero discrepa de ella en la forma de caracterizar la justificación. En el análisis tradicional, la justificación de una proposición $p$ se hace aduciendo otra proposición $q$, o conjunto de proposiciones $K$, que dan un fuerte soporte a $p$. Esto es, las proposiciones en $K$ son las

5 The Central Questions of Philosophy, op. cit., p. 106. 
razones en las que nos basamos o apoyamos para aceptar $p$. La condición de justificación así especificada, según Ayer, da pie a las siguientes objeciones:

i) Excluye la posibilidad de que uno conozca proposiciones para las cuales es inapropiado pedir pruebas.

ii) Excluye la posibilidad de que una persona sepa que $p$ es verdadera si es incapaz de presentar prueba alguna, a pesar de que la pregunta acerca de la evidencia sea pertinente.

iii) Genera un regreso al infinito. ${ }^{6}$

En i) entrarian, por ejemplo, los casos de las sensaciones. Las proposiciones que se refieren a sensaciones como las de dolor, al ser proferidas por una persona, no pueden adecuadamente ser cuestionadas por otra persona, como tampoco tendría sentido que se le pidiera a la persona que expresa dicha proposición, las razones por las que cree saber que tiene dicha sensación. ${ }^{7}$ Los filósofos que aceptan la existencia de proposiciones básicas sostendrían lo mismo con respecto a dichas proposiciones; en el caso de Moore, por ejemplo, no tendría sentido cuestionarse la verdad de proposiciones tales como "ésta es mi mano", proferida al momento de levantar la propia mano; ${ }^{8} 0$ bien, proposiciones proferidas en un contexto normal, o en condiciones estándar, en los casos de Austin ${ }^{9}$ y Quinton. ${ }^{10}$ Podríamos distinguir la tesis de la "incuestionabilidad" de ciertos tipos de proposiciones, en donde es importante el contexto de proferimiento, como en las posiciones de Moore, Austin y Quinton, de a) la tesis, por ejemplo de Descartes, en donde cierto tipo de proposiciones es incuestionable y el contexto de proferimiento es irrelevante, y b) la tesis en donde ciertas proposiciones particulares son proferidas en ciertos contextos, pero en donde carecemos de una regla general que nos permita identificarlas, que sería una de las lecturas de la postura de Wittgenstein.

En ii), Ayer incluye el caso de una persona que consistentemente predice con éxito los resultados de la lotería. ${ }^{11}$ Ayer desecha rápidamente esta objeción diciendo que es un caso dudoso de conocimiento. Sin embargo, Ayer deja fuera de consideración los casos en donde una persona podría muy bien tener conocimiento a pesar de que no pudiera darnos razones y en donde no es tan.

6 Cf. "Knowledge, Belief and Evidence", recogido en Metaphysics and Common Sense, op. cit., pp. 117-118.

7 Wittgenstein va aun más lejos y sostiene que es erróneo anteponer las nociones de conocimiento y creencia a dichas afirmaciones. La afirmación "Yo sé que tengo un dolor" no tendría sentido, como tampoco tendría sentido que la persona negara saber que tiene un dolor. Cf. On Certainty, Oxford: Basil Blackwell, 1969, § 57; § 58.

8 Cf. "Proof of the External World", Proceedings of the British Academy, vol. XXV, 1939.

9 Vide, Sense and Sensibilia, Oxford: Clarendon Press, 1962.

10 Vide, The Nature of Things, London: Routledge and Kegan Paul, 1973.

11 Cf. "Knowledge, Belief and Evidence", op. cit., p. 118. 
claro que, por ello, negaríamos que sabe. Considérense, por ejemplo, proposiciones tales como el saber el nombre de uno mismo o el saber que lo que está frente a mí es un árbol, etc. ${ }^{12} \mathrm{Al}$ igual que Ayer, no me detendré en la consideración de estos casos.

En estas objeciones se presupone que hay que mantener un análisis uniforme del conocimiento para todas las proposiciones; dado este supuesto, la existencia de los casos mencionados en i) y ii) iría en contra del análisis tradicional. Las alternativas que se nos abren son: a) negar que los posibles contraejemplos a la caracterización uniforme son casos legítimos de conocimiento, o b) admitir que son casos legitimos de conocimiento y entonces explicar de qué manera podrían caber dentro de la caracterización general, o c) cuestionarse la idea de que haya un análisis uniforme del conocimiento. Me conformaré con apuntar estas alternativas, sin detenerme a considerar la manera en que Ayer explicaría los diferentes casos, y paso a lo que Ayer dice acerca de la tercera objeción, que a su juicio es una objeción más seria.

La situación regresiva surge porque para que las pruebas presentadas realmente constituyan una base sólida o fuerte para justificar a otra proposición, se requiere que las pruebas mismas sean a su vez conocidas, y así sucesivamente, puesto que de otra manera no habría garantía de que hubiera conocimiento. Asi, escribe Ayer:

Si se me preguntase cómo sé que una proposición $p$ es verdadera, ciertamente serfa insuficiente que yo fuera capaz de presentar otra proposición $q$, aun cuando se diera el caso de que $q$ misma fuera verdadera. Si yo no sé también que $q$ es verdadera, podré haber dado una razón para aceptar $p$, pero no habré dado una razón para aceptar mi afirmación de que sé que $p .{ }^{13}$

Esto es, que para poder justificar la afirmación de que se tiene conocimiento, es necesario que se conozcan todas las proposiciones pertinentes para que se dé la relación de justificación.

Podriamos agregar que el regreso infinito se genera, dada la restricción que se impone a la relación de justificación: la restricción de que las razones deban de garantizar la verdad de la afirmación de que se sabe que $p$. Lo que el regreso infinito demostraría es que si el conocimiento procediera siempre indirectamente, el conocimiento resultaria imposible. De igual manera, si la justificación fuera siempre indirecta y si se piden razones concluyentes, entonces la justificación resultaría imposible. El regreso no se generaría necesariamente si la relación de justificación se especificara de otra manera, sin

12 Vide, Wittgenstein, On Certainty, op. cit., por ejemplo, §§ 327-332; § 425 y $\$ 470$.

13 "Knowledge, Belief and Evidence", op. cit., p. 118. 
tener que renunciar a la tesis de que la justificación es siempre una relación entre proposiciones. Pero éste no es el camino que Ayer sigue.

Ha habido varios intentos para superar la dificultad del regreso, ninguno de ellos, al parecer de Ayer, satisfactorio, en vista de lo cual optará por admitir, por un lado, un tipo de justificación en términos de otros elementos que no son proposiciones $y$, por otro, un estándar de conocimiento menos riguroso que el de otros filósofos. ${ }^{14}$ En la condición de justificación, Ayer sustituye el "dar un fuerte soporte" por el "tener el derecho a estar seguro", en donde lo que da a un individuo el derecho a estar seguro de que $p$, puede ser o bien otra proposición -o proposiciones-, o que el sujeto tenga -o haya tenido- cierta experiencia. ${ }^{15}$ En definitiva, Ayer usa la admisión de diferentes formas de adquirir pruebas como la manera de trazar la línea entre diferentes tipos de proposiciones: las que nos remiten directamente a las experiencias mismas, y que en ese sentido se conocen directamente, y todas las demás proposiciones. Ast, la prueba en el caso de las proposiciones-P, o de las proposiciones-E, si es que se admite la existencia de sense-data, nos remonta a la experiencia de la cual surgió o se originó la creencia, y la prueba en el caso de las otras proposiciones nos remonta a otras proposiciones hasta llegar, en último término, a las proposiciones que se conectan con las experiencias. El tipo de proposición de que se trate determina el tipo de justificación que le corresponde y Ayer no deja abierta la posibilidad de que las proposiciones que se conectan directamente con las experiencias puedan justificarse de otra manera que no sea a través de las experiencias mismas.

Hoy en día, la mayoria de los filósofos sostienen que las cuestiones psicológicas deben de distinguirse de las cuestiones lógicas y epistemológicas. Ayer, sin embargo, no parecería estar estrictamente de acuerdo con esto, por lo menos en lo que respecta a la justificación. Para Ayer una experiencia justifica a una proposición, si la proposición se conecta directamente con la experiencia; la creencia se justifica si se origina directamente en la experiencia. Es apropiado hablar de justificación en el caso de que exista una relación causal directa entre una experiencia y la proposición en cuestión. Así, si se acepta la existencia de sense-data, los enunciados-E serlan los que se justificarían por las experiencias, y en el caso de no aceptarlos, serian los enunciados-P los que se justificarian a partir de las experiencias. Ayer escribe respecto a la relación causal justificativa:

La relación fáctica entre la creencia y la experiencia es lo que justifica mi creencia en la proposición que describe a la experiencia. Esta relación es causal: la filiación de la creencia es compleja. Depende en parte,

14 Ibid., pp. 124-125.

15 lbid., $\mathrm{P} 121$. 
de que me haya formado el hábito de interpretar mis experiencias de cierta manera, pero la existencia de la experiencia es un factor causal.16

Ayer agrega que no es necesario que la persona que tiene la experiencia se percate de que ésta es tal como la describe, ni tampoco de que se percate de que existe una relación causal entre la experiencia y la creencia, para que haya justificación, a pesar de que en la práctica esto normalmente suceda. Para Ayer, si el sujeto tuviera que saber que la descripción de la experiencia es adecuada y tuviera que saber que la experiencia está causalmente conectada a ella, se volvería a generar un regreso al infinito. Por tanto, la justificación de la creencia la provee directamente la experiencia. ${ }^{17}$ Sin embargo, Ayer tiene que admitir que el sujeto, de alguna manera, se da cuenta de la experiencia y que, de hecho, si el sujeto tiene la creencia, debe de haber tenido la experiencia aun cuando no la presente como la razón por la cual acepta la creencia; la experiencia es su razón aun cuando el sujeto no sea plenamente consciente de ella y sin importar que el sujeto la presente o no como su razón.

Ya vimos en páginas anteriores que Ayer también sostiene que hay una relación causal entre los enunciados-E y los enunciados-P. Sin embargo, no considera que esta relación sea la que proporcione la justificación del enunciado-P. Ayer dice explícitamente (recuérdese la cita 5) que la justificación, en este caso, la provee la constancia y coherencia que presentan nuestras experiencias, y que el enunciado-P es la mejor forma de organizar o explicar nuestras experiencias. Ayer no explica en ningún escrito por qué la relación causal no sería justificativa $o$, por lo menos, que no seria justificativa por sf sola, de los enunciados-P.

Es engañoso cómo Ayer presenta las dos formas de justificación, puesto que nos hace suponer que las dos formas son mutuamente excluyentes. Sin embargo, para darle plausibilidad a su propia postura, hay que admitir que los enunciados-P se justifican tanto por la experiencia como por otras proposiciones. La razón por la que la relación causal en este caso no serfa suficiente, serfa porque lleva implícita una ontologia, y si sólo consideráramos la relación causal, nunca se justificarfan los enunciados-P. La tesis de la ontologfa implícita en los enunciados-P también es la razón por la cual Ayer cree que la verdad de los enunciados-E nunca podrían garantizar la verdad del enunciado-P.

Aun cuando admitiésemos que hay una conexión directa entre las experiencias y ciertas proposiciones, esto no nos compromete a decir que la relación que se mantiene entre aquélla y éstas sea una relación de justificaciơn; se tendría que explicar cómo se legitima el que tomemos a la experiencia

18 lbid., p 123.

17 Ibid., p 123. 
como la razón para aceptar la proposición. Podríamos muy bien aceptar que la experiencia causa o genera ciertas creencias, sin tener que admitir que las justifica. El temor de Ayer es que si las experiencias no justificaran, nuestras afirmaciones de creencia y de conocimiento se quedarian injustificadas, además de la amenaza del regreso al infinito. Estos temores desaparecerfan si creyéramos, como cree Wittgenstein, que la base para aceptar ciertas creencias está más allá de toda justificación; éstas serian las creencias que son transmitidas implícitamente en nuestro aprendizaje lingüístico. Para Wittgenstein, el hecho de que ciertas proposiciones no necesiten justificarse, se puede apreciar si reflexionamos en la manera en que las adquirimos. El contraste que quiere establecer Wittgenstein es entre las proposiciones que aceptamos porque estamos convencidos de su verdad, esto es, porque poseemos razones convincentes, y las proposiciones que aceptamos porque nos fueron heredadas. Lo que caracteriza, por lo menos, a algunas de éstas últimas, es que si las cuestionáramos, cuestionaríamos también nuestra comprensión de ellas. ${ }^{18}$ Algunas de estas ideas las sintetiza Wittgenstein en los siguientes pasajes:

128. Asf aprendi a juzgar desde niño. Esto es juzgar.

129. Así es como aprendí a juzgar; a conocer que esto es un juicio.

130. Pero, ¿no es acaso la experiencia lo que nos enseña a juzgar asi, esto es, que es correcto juzgar de esta manera? Nosotros podremos extraerlo de la experiencia, pero la experiencia no nos guia para extraer algo de ella. Si es la base (o fundamento) para que juzguemos así, y no sólo la causa, de todas formas, todavía no tenemos la base para ver esto, a su vez, como una base.

131. No, la experiencia no es el fundamento de nuestro juego para juzgar. Tampoco lo es su éxito sobresaliente.

144. El niño aprende a creer muchas cosas, esto es, aprende a actuar de acuerdo con estas creencias. Poco a poco se forma un sistema de lo que se cree, y en este sistema, algunas cosas se mantienen firmes y otras están más o menos abiertas a modificación. Lo que se mantiene estable (o firme), no se debe a que sea obvio o convincente intrínsecamente, sino más bien lo mantiene firme lo que está a su alrededor. ${ }^{10}$

En conclusión, podríamos decir que la maniobra de Ayer no le permite escapar del regreso infinito, a no ser que lograra explicar de qué manera es posible establecer una relación de justificación entre ciertas proposiciones y elementos extra-lingüisticos, y en donde la relación de justificación no presuponga que el sujeto tenga otras creencias.

En general se ha creído que las proposiciones requieren de justificación,

18 On Certainty, § 94; $1140 ; \S 880-83$.

19 Ibid. 
por el hecho de que son inferenciales. Ayer intenta romper esta conexión entre justificación e inferencialidad y mantener que aun en los casos en donde no haya inferencia es pertinente preguntar por la justificación. Pero el que tenga sentido preguntarse por la justificación de las creencias no-inferenciales, es una cuestión abierta a discusión.

Ayer dice que la plausibilidad de su programa descansa en la tesis acerca de la justificación ${ }^{20}$ y si tiene razón al decir esto y esta tesis resulta insatisfactoria, entonces habrá perdido la última motivación para seguirse aferrando a la existencia de sense-data. Cada intento de Ayer por defender su posición empirista de sense-data, lo ha llevado a poner, él mismo, la soga al cuello a la tesis de sense-data, ya que los mismos hechos que él admite son susceptibles de explicación sin necesidad de hablar de inferencias; más aún, hablar de inferencias - justificativas o no- serfa erróneo.

Salma SaAb

Instituto de Investigaciones filosóficas

Universidad Nacional Autónoma de México

20 Ayer sostiene que su proyecto de construcción del mundo físico es viable, siempre y cuando no se pretenda que el lenguaje de sense-data tenga una prioridad, ya sea ontológica - epistemológica, frente al lenguaje que se refiere a objetos físicos. No se trata de una prioridad ontológica, ya que son ontológicamente neutrales, y no es una prioridad epistemológica, ya que no se busca que el lenguaje de sense-data tenga que ser nuestro lenguaje primitivo, en relación al lenguaje que se refiere a objetos materiales. Cf. The Central Questions of Philosophy, op. cit., p. 90 y p. 108. 